\title{
UV and X-ray observations of the neutron star LMXB EXO 0748-676 in its quiescent state
}

\author{
A.S. Parikh, ${ }^{1}$ N. Degenaar, ${ }^{1 \star ~ J . V . ~ H e r n a ́ n d e z ~ S a n t i s t e b a n, ~}{ }^{1,2}$ R. Wijnands, ${ }^{1}$ \\ I. Psaradaki, ${ }^{1,3}$ E. Costantini, ${ }^{1,3}$ D. Modiano, ${ }^{1}$ and J.M. Miller ${ }^{4}$ \\ ${ }^{1}$ Anton Pannekoek Institute for Astronomy, University of Amsterdam, Science Park 904, NL-1098 XH Amsterdam, the Netherlands \\ ${ }^{2}$ School of Physics and Astronomy, University of St. Andrews, North Haugh, St. Andrews, Fife, KY16 933, Scotland, UK \\ ${ }^{3}$ SRON Netherlands Institute for Space Research, Sorbonnelaan 2, 3584 CA Utrecht, The Netherlands \\ ${ }^{4}$ Department of Astronomy, University of Michigan, 1085 South University Avenue, Ann Arbor, MI 48109, USA
}

Accepted 2020 November 26. Received 2020 November 26; in original form 2020 June 26

\begin{abstract}
The accretion behaviour in low-mass X-ray binaries (LMXBs) at low luminosities, especially at $<10^{34} \mathrm{erg} \mathrm{s}^{-1}$, is not well known. This is an important regime to study to obtain a complete understanding of the accretion process in LMXBs, and to determine if systems that host neutron stars with accretion-heated crusts can be used probe the physics of dense matter (which requires their quiescent thermal emission to be uncontaminated by residual accretion). Here we examine ultraviolet (UV) and X-ray data obtained when EXO 0748-676, a crust-cooling source, was in quiescence. Our Hubble Space Telescope spectroscopy observations do not detect the far-UV continuum emission, but do reveal one strong emission line, C IV. The line is relatively broad $\left(\gtrsim 3500 \mathrm{~km} \mathrm{~s}^{-1}\right)$, which could indicate that it results from an outflow such as a pulsar wind. By studying several epochs of X-ray and near-UV data obtained with XMM-Newton, we find no clear indication that the emission in the two wavebands is connected. Moreover, the luminosity ratio of $L_{\mathrm{X}} / L_{\mathrm{UV}} \gtrsim 100$ is much higher than that observed from neutron star LMXBs that exhibit low-level accretion in quiescence. Taken together, this suggests that the UV and X-ray emission of EXO 0748-676 may have different origins, and that thermal emission from crust-cooling of the neutron star, rather than ongoing low-level accretion, may be dominating the observed quiescent X-ray flux evolution of this LMXB.
\end{abstract}

Key words: accretion, accretion discs - stars: neutron stars - ultraviolet: general - X-rays: binaries

\section{INTRODUCTION}

Low-mass X-ray binaries (LMXBs) are systems wherein a compact object accretes matter from a companion star, typically via an accretion disc. Many LMXBs exhibit transient behaviour and for these sources the accretion of matter onto the compact object is expected to (almost) stop during quiescence (e.g., Lasota 2001; Hameury 2020). The outburst luminosities exhibited by transient LMXBs hosting neutron stars (NSs) are typically on the order of $10^{35}-10^{38} \mathrm{erg} \mathrm{s}^{-1}$ in the $0.5-10 \mathrm{keV}$ energy range (e.g., Yan \& Yu 2015). In transient systems these luminosities drop to levels of $10^{30}-10^{34} \mathrm{erg} \mathrm{s}^{-1}$ when the outbursts cease (see e.g., Wijnands et al. 2017, for a review).

During the brightest phases of an outburst, typically at luminosities $\gtrsim 10^{36} \mathrm{erg} \mathrm{s}^{-1}$, the accretion flow takes on the form of a geometrically thin, optically thick disc (e.g., Shakura \& Sunyaev 1973; Lasota 2001). The disc may be accompanied by a corona, a Comptonising medium of hot electrons, likely located close to the central compact object (e.g., Done et al. 2007; Gilfanov 2010). The properties of the accretion flow close to the compact object are thought to change significantly towards lower luminosities by transitioning into a geometrically thick, optically thin flow also referred to as a radiatively-inefficient accretion flow (RIAF; e.g., Dubus et al. 2001;

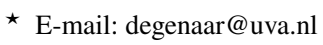

Narayan \& Yi 1994). However, the conditions at which the transition from a standard disc to a RIAF take place are not very well understood, and hardly constrained by observations.

Studies of quiescent accretion flows are further exacerbated, as the inherent low luminosities exhibited in this state make it difficult to obtain high-quality data. The mere presence and properties of a disc, corona and/or RIAF at these luminosities is not definitively known (e.g. Hynes \& Haswell 1999; Hynes et al. 2005; Cackett et al. 2013a), so it is not clear if, and how, accretion takes place. Accretion onto the compact object could potentially come to a halt in quiescence, but there could also be ongoing inward motion of the material through a disc/corona/RIAF, perhaps still enabling lowlevel accretion onto the compact object. Studies in quiescence are important to understand accretion at low luminosities and contribute to our overall understanding of accretion processes in LMXBs.

Apart from studying accretion, transient NS LMXB systems can also be used as laboratories to probe the properties of dense matter present in NS crusts and cores (e.g., Brown et al. 1998; Cumming et al. 2017; Wijnands et al. 2017). During outbursts, the accreted matter compresses the NS crust and induces reactions such as electron captures, neutron emissions, and density-driven fusion reactions (e.g., Haensel \& Zdunik 1990, 2008; Steiner 2012). These reactions release heat in the crust, disrupting its thermal equilibrium with the core. Furthermore, observations of NS LMXBs have indicated that, 
in addition to these theoretically predicted heating reactions, heat may also be released at shallow depths in the crust by an unknown 'shallow heating' process (e.g., Brown \& Cumming 2009; Degenaar et al. 2011b, 2014).

With the cessation of an outburst, i.e., active accretion, the compression-induced heating reactions occurring in the NS crust are expected to stop. As a result, in quiescence the crust cools to reinstate the thermal equilibrium with the core. Monitoring this cooling evolution and comparing it to theoretical models, has been used to investigate the behaviour of the dense matter present in NS crusts (e.g., Wijnands et al. 2001; Rutledge et al. 2001; Shternin et al. 2007; Brown \& Cumming 2009; Page \& Reddy 2013; Turlione, Aguilera, \& Pons 2015; Ootes et al. 2018). So far, such crust-cooling studies have been carried out for ten NSs in LMXBs by examining their X-ray spectral evolution (see Wijnands et al. 2017, for a review). The quiescent spectra of NSs exhibiting crust cooling are typically characterised by a prominent thermal emission component (often contributing $>70$ per cent of the total $0.5-10 \mathrm{keV}$ flux) that is interpreted as heat radiation from the NS surface.

For several NSs in LMXBs there is evidence that there is some form of low-level accretion occurring, either continuously or in spurts, at similarly low luminosities as are exhibited by crust-cooling sources in quiescence (e.g., EXO 1745-248, Cen X-4, Aql X-1, XTE J1701462; Fridriksson et al. 2011; Degenaar \& Wijnands 2012; Bernardini et al. 2013a; Coti Zelati et al. 2014a). Notably, the spectra of these sources are characterised by a prominent power-law shaped emission component that, when detailed studies are possible, appears to extend to energies $>10 \mathrm{keV}$ (Chakrabarty et al. 2014). Often this power-law emission component occurs alongside a thermal emission component. Indeed, theoretical calculations suggest that low-level accretion onto a NS can produce a composite spectrum of thermal and power-law like emission components (e.g., Deufel, Dullemond, \& Spruit 2001).

Several crust-cooling sources also require a power-law shaped component, in addition to thermal surface emission, to adequately fit their quiescent spectra (e.g., Degenaar et al. 2009a; Wijnands et al. 2015; Parikh et al. 2017). This could potentially indicate that lowlevel accretion is ongoing in these systems. If true, it is not trivial to disentangle contributions of low-level accretion and crust-cooling emission to the thermal emission spectra. This then complicates our determination of the crust-cooling evolution and thereby the inferred NS physics. Constraining the properties of low-level accretion in LMXBs is therefore also important to correctly infer the physics of the NS crust and core.

In addition to examining the X-ray spectra, accretion can also be probed at ultraviolet (UV) wavelengths. The surface temperatures of NSs in LMXBs are generally such $\left(\simeq 10^{6}-10^{7} \mathrm{~K}\right)$ that their cooling emission is not expected to significantly contribute to their UV emission. Also, the inherent emission from the late-type companion star contributes negligibly to the UV emission of the system. However, the accretion stream, arising from the Roche lobe overflow of the donor star, is expected to be UV bright, hence making the UV a suitable wavelength regime to probe the quiescent accretion flows. Previous studies of quiescent LMXBs have indicated that the UV emission arises from small emitting radii with unexpected high temperatures, suggesting that this does not come from the bulk of the disc but rather from the impact point of matter from the companion star hitting the accretion disc (e.g., Froning et al. 2011), or X-ray irradiation of the inner accretion disc or companion star (e.g., Hynes \& Robinson 2012; Cackett et al. 2013a; Bernardini et al. 2013a).

A further indicator of possible ongoing accretion towards the NS can be derived from the correlation exhibited between the X-ray and
UV luminosities in quiescence (e.g., Hynes \& Robinson 2012). In particular, if the UV and X-ray luminosities are similarly bright, the UV emission is too high to only arise from irradiation of the disc (if present) and/or the companion star by X-rays from the central compact object (or from a hot inner accretion disc, if present).

Most NS LMXBs cannot be studied in the UV as they exhibit strong interstellar extinction along the line of sight. However, a few LMXBs exhibit less strong extinction, allowing us to probe for signatures of low-level accretion in the UV. One such source is the crust-cooling source EXO 0748-676.

\subsection{The NS LMXB EXO 0748-676}

The NS LMXB EXO 0748-676 (UY Vol) was discovered almost three decades ago (Parmar et al. 1985). It exhibited X-ray eclipses (lasting $\simeq 500 \mathrm{~s}$ ), indicating that the binary is viewed at high inclination $\left(i \simeq 75^{\circ}-83^{\circ}\right)$. Its orbital period was determined to be $\simeq 3.8 \mathrm{hr}$ (Parmar et al. 1986; Wolff et al. 2009). The source displayed thermonuclear X-ray bursts, which indicated the NS nature of the primary and resulted in a distance estimate of $\simeq 7.4 \mathrm{kpc}$ (Wolff et al. 2005).

EXO 0748-676 exhibited an accretion outburst that is estimated to have started between 1980 and 1984 (Degenaar et al. 2009a). It remained active until September 2008 (Wolff et al. 2008a,b; Hynes \& Jones 2008; Torres et al. 2008). Observations of the source in quiescence indicate that the crust of the NS was heated up during outburst and exhibited cooling in quiescence (e.g., Degenaar et al. 2009a, 2011a, 2014; Díaz Trigo et al. 2011; Cheng et al. 2017). However, more recent quiescent observations, obtained $>2000 \mathrm{~d}$ after the end of its accretion outburst, indicate a rise in the observed effective NS temperature (Parikh et al. 2020). This rise is highly surprising for a crust-cooling source as it is expected to exhibit a continuous decay in the temperature rather than a rise. In this work, we investigate if low-level accretion occurs in the quiescent state of EXO 0748-676.

\section{OBSERVATIONS AND DATA ANALYSIS}

We examine UV spectroscopic and imaging data of EXO 0748-676. We also compare these UV data with (quasi-)simultaneous X-ray data. De-reddened photometric fluxes and magnitudes were calculated by assuming $E(B-V)=0.06$ (Hynes et al. 2006) and the Galactic extinction curves of Cardelli et al. (1989). The de-reddening has been carried out in Python using the ExTINCTION package. ${ }^{1}$ All uncertainties reflect $1 \sigma$ confidence levels.

\subsection{Hubble Space Telescope observations}

\subsubsection{Far-UV spectroscopy}

We obtained far-UV (FUV) spectroscopic observations of EXO 0748-676 in quiescence using the Cosmic Origins Spectrograph (COS; Green et al. 2012) on board the Hubble Space Telescope (HST) under the GO program 13108 (PI: Degenaar). The source was targeted for 9 orbits divided over 2 visits, starting on 2013 March 28 (MJD 56379). The total on-source exposure time amounted to $\simeq 7.4 \mathrm{hr}$. The COS was operated using the low-resolution G140L grating with segment B switched off. This provided FUV coverage in

\footnotetext{
1 https://extinction.readthedocs.io/en/latest/
} 
the wavelength range of $\simeq 1118-2251 \AA$ with a resolving power of $\lambda / \Delta \lambda \simeq 2500-3500 \AA$. For each exposure, the grating was stepped through different fp-pos positions to minimize the fixed pattern noise. All the data were taken in TIME-TAG mode. These COS data were retrieved from the Multi-Mission Archive at $\mathrm{STScI}^{2}$. The downloaded data have been pre-processed with CALCOs (version 3.3.7).

To contextualise the quiescent FUV spectra of EXO 0748-676, we compared it to the FUV spectrum obtained during outburst. This outburst spectrum was obtained in 2003 February 18 (MJD 52688) using the Space Telescope Imaging Spectrograph (STIS; e.g., Ely et al. 2015). The source was observed for $\simeq 9 \mathrm{hr}$ over 2 complete orbits in the continuous viewing zone. The observations were carried out using the FUV MAMA detector (Joseph et al. 1995) and the G140L grating. The data were taken in the TIME-TAG mode. The STIS data were retrieved from the Multi-Mission Archive at STScI and were pre-processed with CALSTIS (version 3.4.2). Further details of these data are provided in Hynes et al. (2006) and Pearson et al. (2006).

Spectral analysis on these FUV data was performed on the products downloaded from the archive. The various one-dimensional spectra, corresponding to each quiescent epoch, were combined using the SPLICE task in $\operatorname{IRAF}^{3}$ (e.g., Tody 1986). These spectra were further analysed using the sPECUTILs package ${ }^{4}$ (Astropy-Specutils Development Team 2019) in Pyтноn and are shown in Figure 1. Line widths were calculated based on Gaussian fits using LINE_FLuX, part of the SPECUTILs package in Python.

\subsubsection{Near-UV photometry}

Each of the two HST visits of EXO 0748-676 during quiescence were preceded by acquisition imaging observations of $51 \mathrm{~s}$ each, using mirror A and the PSA aperture. This provided sensitivity in the UV range of $\simeq 1600-3300 \AA$, peaking at $\simeq 2300 \AA$.

We estimated a Near-UV (NUV) ${ }^{5}$ magnitude for EXO 0748-676 from these acquisition images. Source events were extracted from an aperture of radius $0.10^{\prime \prime}$ using РyтHON. Furthermore, an aperture of three times that size was used to estimate the background. The detected count rates were converted to fluxes using the count rate to flux conversion factor appropriate for our instrumental set-up. This conversion factor, reported in the headers of the acquisition images, was found to be 1 counts $\mathrm{s}^{-1}=4.82 \times 10^{-18} \mathrm{erg} \mathrm{cm}^{-2} \mathrm{~s}^{-1} \AA^{-1}$.

\subsection{Swift observations}

EXO 0748-676 was observed with the X-ray telescope (XRT; Burrows et al. 2005) on board the Neil Gehrels Swift Observatory (Gehrels et al. 2004). A $\simeq 7.8 \mathrm{ks}$ observation was carried out on 2013 March 23 (MJD 56374), i.e., 5 d before the HST UV observations were obtained. We reduced the Swift data $^{6}$ using the HeAsoft package (version 6.13), by re-processing the data using the XRTPIPELINE. We extracted the spectrum of the source with XSELECT

\footnotetext{
2 https://archive.stsci.edu/hst/search.php

3 https://iraf-community.github.io/install.html

4 https://specutils.readthedocs.io/en/stable/index. html

5 In this paper, we define NUV to encompass all data in the wavelength range $1600-3000 \AA$ A. These include the NUV data discussed in Sections 2.1.2, 2.2, and 2.3.

6 Obtained from the Swift archive, found at https://heasarc.gsfc. nasa.gov/cgi-bin/w3Browse/swift.pl
}

using a circular region with a radius of $35^{\prime \prime}$ centred on EXO 0748676. A surrounding annular region, having an inner and outer radius of $100^{\prime \prime}$ and $200^{\prime \prime}$ respectively, was used to obtain a background spectrum. The spectral data were grouped into bins with a minimum of 10 photons per bin using the Heasoft tool GRPPHA. The spectrum was fit using XSPEC.

Since EXO 0748-676 hosts a cooling NS crust (e.g., Parikh et al. 2020, see the references therein for more details), we fitted the XRT spectrum with NSATMOS (a NS atmosphere model; Heinke et al. 2006). The source distance was set to $D=7.4 \mathrm{kpc}$ (e.g., Galloway et al. 2008) and the NS mass and radius to $M=1.6 \mathrm{M}_{\odot}$ and $R=$ $12 \mathrm{~km}$, respectively. Given the limited statistics of the XRT data, we fixed several of the NSATMOS parameters to the values obtained from fitting high-quality quiescent Chandra and XMM-Newton spectra of EXO 0748-676 (see Parikh et al. 2020, for details, where the same parameters have been fixed). An additional power-law component was needed to fit some high-quality quiescent spectra of this source (Parikh et al. 2020). Thus, we also use this component for the XRT spectral fitting here. The power-law index was fixed to $\Gamma=1.0$ and the normalisation was free to vary. In addition, we fix the column density to $N_{\mathrm{H}}=4.3 \times 10^{20} \mathrm{~cm}^{-2}$, as determined from fitting high quality data, presented in Parikh et al. (2020).

Alongside the XRT, Swift hosts the Ultraviolet Optical Telescope (UVOT; Roming et al. 2005), with NUV photometric and spectroscopic capabilities. We examined all archival UVOT data. We processed the data using the uvousource tool. However, the source was not detected by the UVOT during any quiescent observations. Furthermore, since the upper limits obtained from these data were not constraining we do not discuss them further.

\subsection{XMM-Newton observations}

The X-ray Multi-Mirror Mission or XMM-Newton hosts instruments with observation capabilities in the X-ray and NUV wavelength regimes. This allows for correlated X-ray-UV studies as instruments observing in both wavelength ranges operate simultaneously. So far, EXO 0748-676 has been observed 6 times during quiescence using XMM-Newton. These observations were downloaded from the XMM-Newton archive. ${ }^{7}$

The X-ray data, obtained using the European Photon Imaging Camera (Strüder et al. 2001; Turner et al. 2001), were processed by extracting the spectra and fitting them collectively in XSPEC. The details of the spectral extraction and spectral fitting are provided in Section 2 of Parikh et al. (2020).

The NUV photometric data, obtained using the Optical Monitor (OM; Mason et al. 2001), were only available for five of the six observations. This is because the data for observation 3 (corresponding to observation Id 0605560501) were not obtained due to a technical error (Díaz Trigo et al. 2011). For the available data, the NUV fluxes were extracted using the OM tasks OMICHAIN and OMFCHAIN in SAS, as was appropriate when the photometric data were obtained in the imaging mode and in the fast mode, respectively. We examined the fluxes corresponding to the full exposure (for each available filter, from the output files produced by OMICHAIN and OMFCHAIN) from each entire observation, in order to use the most constraining flux estimate for a given epoch. Furthermore, this is because we do not expect to be able to detect any orbital variations in the flux due to the low UV luminosities exhibited by EXO 0748-676 (as was shown

\footnotetext{
7 http://nxsa.esac.esa.int/nxsa-web/\#home
} 
by Díaz Trigo et al. 2011, during the earliest quiescent UV observations obtained). EXO 0748-676 was not detected during several OM observations (see Table 1). In case of non-detections, we used the faintest detected source in the associated observation as an upper limit estimate for EXO 0748-676 (see Table 1). This was done by examining the output files produced by омісHAIN and oMfCHAIN. Since the default detection threshold is $3 \sigma$, our obtained upper limits correspond to this significance level.

\section{RESULTS}

\subsection{The HST FUV spectra}

\subsubsection{Quiescent spectrum}

Despite the low extinction towards EXO 0748-676, the quality of the quiescent FUV spectra obtained by $H S T$ during individual orbits was low. This was due to the inherently low source flux in quiescence. The signal-to-noise ratio was inadequate to perform any phase-resolved spectral analysis which is necessary to investigate signatures of the orbital motion. We, therefore, combined individual exposures into a uniform wavelength grid (with $\Delta \lambda=5 \AA$ ) and applied an error-weighted average using the sPECTRES package (Carnall 2017) in Python. The resulting averaged spectrum is shown in red in Figure 1. Although we combined the different COS exposures, the resulting spectrum was still of low signal-to-noise and there was no continuum emission detected above $\simeq 10^{-17} \mathrm{erg} \mathrm{cm}^{-2} \mathrm{~s}^{-1} \AA^{-1}$, preventing us from obtaining any continuum flux measurement from the FUV spectral data.

\subsubsection{Outburst spectrum}

We aim to qualitatively compare the outburst spectrum to that obtained during quiescence and are not interested in probing the orbital variations observed during the outburst. Thus, the individual STIS spectra were combined into one spectrum and as a result, this blurs out the orbital variations observed in outburst (Pearson et al. 2006). This has been done as described in Section 2.1.1. To ensure direct comparison with the quiescent spectrum, the combined outburst spectrum was re-binned to the same resolution (of $\Delta \lambda=5 \AA$ ) as that of the combined quiescent spectrum. This is shown in Figure 1, in black. The dotted, green, vertical lines indicate the significant emission lines in the outburst spectrum, as identified by Pearson et al. (2006). The emission observed near $1580 \AA$ is an artefact and does not correspond to any line (Pearson et al. 2006).

\subsubsection{Airglow observed by the COS instrument}

Airglow is the contribution of emission from the Earth's atmosphere. The quiescent FUV spectrum is shown alongside an example of a spectrum obtained using the same COS instrument when the terrestrial airglow was strongly observed. ${ }^{8}$ This is shown in blue in Figure 1 and has been re-binned to have the same spectral resolution as the quiescent source spectrum. As can be seen, this airglow spectrum roughly matches the strong emission lines seen around 1200, 1215,

\footnotetext{
8 The different observations of airglow by the HST/COS can be found at https://www.stsci.edu/hst/instrumentation/ cos/calibration/airglow. We show the airglow spectrum corresponding to the dataset LBW3E4060, observed on 2012 August 10 (MJD 56149).
}

and $1304 \AA$ in our quiescent spectrum of EXO 0748-676. Evidence from other airglow observations, obtained using COS, show that the emission line observed around $1356 \AA$ is also a result of airglow (e.g., Froning et al. 2014). The prominent wavelengths at which airglow emission lines are expected is shown using vertical, dashed, blue lines. The outburst spectra were taken with a different instrument and do not suffer from airglow (Pearson et al. 2006).

Discarding any emission lines due to airglow, we find that our quiescent observation of EXO 0748-676 only exhibits one significant emission line around $1550 \AA$, identified to be the $C_{\text {IV }}$ line by Pearson et al. (2006). Due to the lack of a continuum detection only the lower limit of the $C_{\text {IV }}$ line could be constrained during quiescence. This

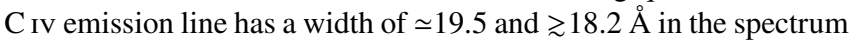
obtained during outburst and quiescence, respectively.

We also investigated if any other lines that were significant during the outburst were present in quiescence. The red wing of the emission line in the quiescent spectra, observed around $1220 \AA$, exhibits an excess as compared to the corresponding airglow emission line. This may be indicative of a $\mathrm{Nv}$ emission line from the source, as was seen during outburst (see Figure 1; see also Pearson et al. 2006). This excess is relative to our choice of the example airglow emission spectrum shown. However, it is not trivial to constrain any excess in the spectrum due to emission by the $\mathrm{N} v$ line in quiescence.

\subsection{The ratio of the X-ray and UV luminosities}

We examined quasi-simultaneous epochs of X-ray and NUV data to investigate the correlation between emission in these two energy bands.

\subsubsection{HST NUV photometry}

Visual inspection of the NUV acquisition images obtained by the HST/COS show that EXO 0748-676 was faintly detectable. Examining these images, we estimated an average (background-subtracted) source count rate of $\simeq 0.16$ counts $\mathrm{s}^{-1}$. Using the appropriate count rate to flux conversion factor (see Section 2.1.2), we estimated a flux density of $F_{\lambda} \simeq 7.3 \times 10^{-19} \mathrm{erg} \mathrm{cm}^{-2} \mathrm{~s}^{-1} \AA^{-1}$, and an $\mathrm{AB}$ magnitude of $\simeq 26.1 \mathrm{mag}$. The de-reddened flux density and magnitude were found to be $F_{\lambda} \simeq 1.2 \times 10^{-18} \mathrm{erg} \mathrm{cm}^{-2} \mathrm{~s}^{-1} \AA^{-1}$ and $\simeq 25.6 \mathrm{mag}$, respectively.

By multiplying the flux with the central wavelength of the instrument passband (2300 ̊; Holland et al. 2012), we estimate a de-reddened UV flux of $F_{U V} \simeq 2.8 \times 10^{-15} \mathrm{erg} \mathrm{cm}^{-2} \mathrm{~s}^{-1}$. For a distance of $7.4 \mathrm{kpc}$, this translates into a luminosity of $L_{\mathrm{UV}} \simeq 1.8 \times 10^{31} \mathrm{erg} \mathrm{s}^{-1}$. We note, however, that this approach effectively assumes that the UV spectrum is flat over the instrument passband, which is not necessarily the case. We investigate the effect of this assumption in Section 3.2.4.

\subsubsection{Swift X-ray data}

The model described in Section 2.2 provided a good description of the X-ray spectral data $\left(\chi_{v}^{2}=0.97\right.$ for 31 d.o.f.). We obtained $k T_{\text {eff }}^{\infty}=111.3 \pm 2.0 \mathrm{eV}$ and the resulting unabsorbed flux was $F_{\mathrm{X}}=$ $(5.3 \pm 0.4) \times 10^{-13} \mathrm{erg} \mathrm{cm}^{-2} \mathrm{~s}^{-1}$, in the $0.5-10 \mathrm{keV}$ energy range. At a distance of $7.4 \mathrm{kpc}$, this corresponds to a luminosity of $L_{X}=(3.5 \pm$ $0.3) \times 10^{33} \mathrm{erg} \mathrm{s}^{-1}$. Thus, using our quiescent quasi-simultaneous Swift X-ray and $H S T$ NUV observations, we obtained a luminosity ratio of $L_{\mathrm{X}} / L_{\mathrm{UV}} \simeq 200$. 


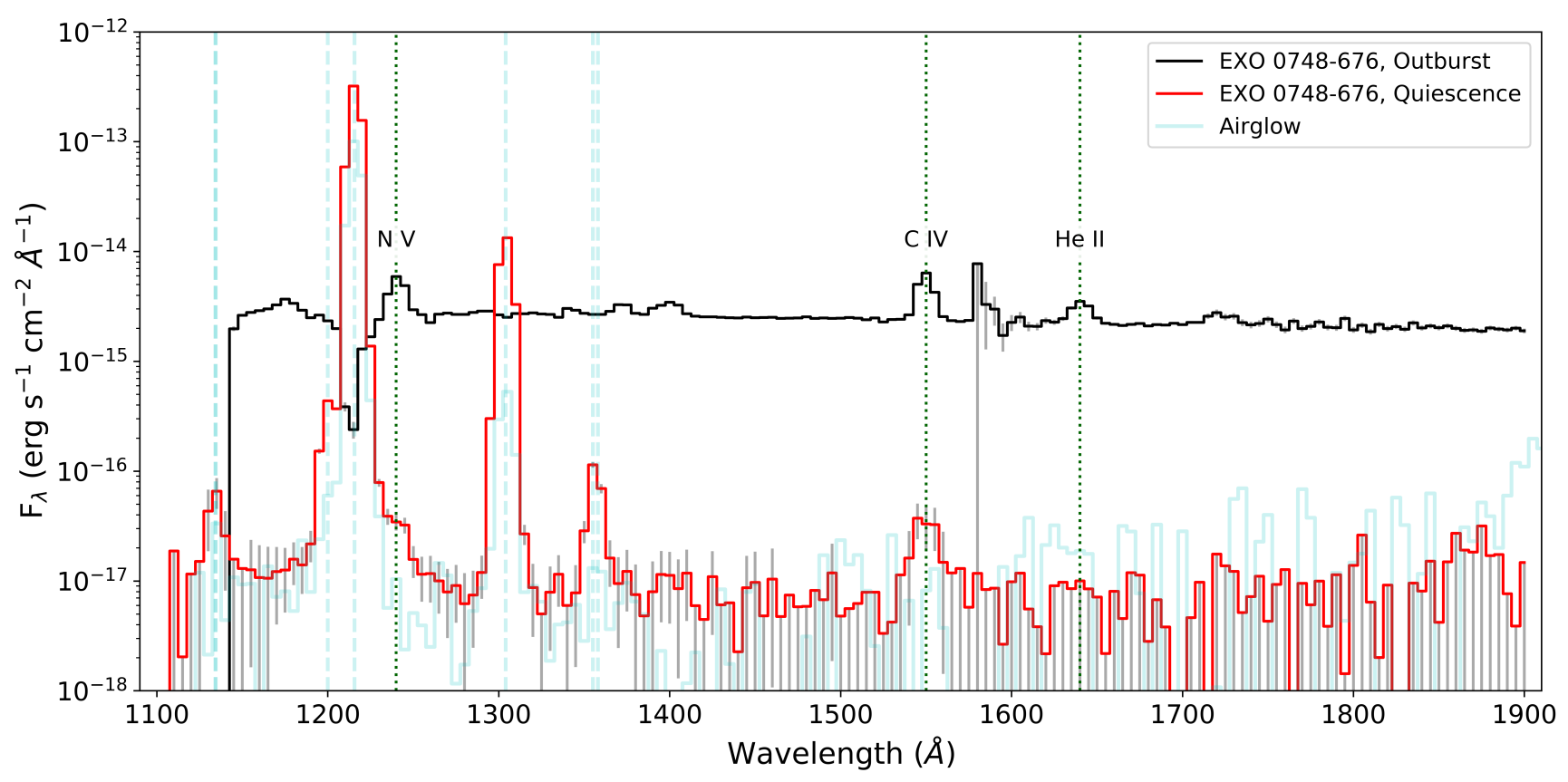

Figure 1. Co-added FUV spectra of EXO 0748-676 obtained using HST during two epochs — during outburst (using STIS, black) and during quiescence (using COS, red). The respective error bars are shown in grey. Both spectra have been re-binned to the same spectral resolution (of $\Delta \lambda=5 \AA$ ). The vertical, dotted, green lines indicate the strong emission lines seen in the outburst spectra, as identified by Pearson et al. (2006). The spectrum shown in blue is an example of the airglow spectrum as observed using the COS instrument. The vertical, dashed, blue lines are often-observed airglow emission lines exhibited during observations obtained using COS (e.g., Froning et al. 2014). The STIS observation does not suffer from contamination due to airglow.

\subsubsection{XMM-Newton X-ray and UV data}

EXO 0748-676 was detected two times in quiescence using the OM with the U filter (see Table 1). Upper limits were obtained during three other epochs - once using the UVW1 filter and twice using the $\mathrm{U}$ filter. The results of the OM photometry is listed in Table 1. The source was detected in X-ray during all the XMM-Newton epochs reported here (i.e., those with accompanying OM observations, see Section 2.3). Details about the X-ray luminosities are presented in Parikh et al. (2020, see their table 1).

If we calculate the UV luminosity by multiplying the inferred UV fluxes with the filter width (but see Section 3.2.4), we obtain $L_{X} / L_{U V}$ ratios of $\simeq 100$ from the $X M M$-Newton data (see Table 1). This is on the same order of magnitude as the ratio inferred for the epoch of quasi-simultaneous HST and Swift observations (see Section 3.2.2).

\subsubsection{The effect of the unknown UV spectral shape}

In Section 3.2.1 and 3.2.3, we estimated $\lambda L_{\lambda}$ for all UV observations in order to compare the X-ray and UV luminosity ratio of EXO 0748-676 with that of the eight LMXBs studied in Hynes \& Robinson (2012). However, the UV spectrum is likely not flat and may significantly change across the wavelength range that we study. To probe the magnitude of this effect, we folded a stellar atmosphere spectra with appropriate effective temperatures (Allard et al. 2012) ${ }^{9}$ through the relevant UV filter and then determined the expected ratio

9 Available at https://phoenix.ens-lyon.fr/Grids/BT-Settl/ of fluxes in different wavebands for each observation. ${ }^{10}$ For this exercise we used two different temperatures, of $5000 \mathrm{~K}$ and $13000 \mathrm{~K}$, based on the UV-optical spectral energy distributions (SEDs) of the eight quiescent LMXBs studied in Hynes \& Robinson (2012). We scaled the two different SEDs by performing synthetic photometry on them and comparing them to the unabsorbed fluxes from Table 1. We then integrated the fluxes and compared these to a flat spectrum (i.e., the implicit assumption when calculating $\lambda L_{\lambda}$ ).

We show the results of these calculations for each observation in Figure A1. The SED shape has the largest effect on the UV luminosity calculation for the bluest filter. For the HST acquisition image, the ratio of the integrated flux from the reddest SED (i.e., the one with an effective temperature of $5000 \mathrm{~K}$ ) compared to a flat spectrum is a factor $\simeq 1.5$. The magnitude of the effect in the $U$ band is much smaller, a few per cent at most (see Figure A1).

\subsection{5 $L_{\mathrm{X}}$ versus $L_{\mathrm{UV}}$}

The unabsorbed X-ray luminosities in the $0.5-10 \mathrm{keV}$ energy range, along with the (quasi-)simultaneously obtained UV luminosities (corrected for extinction) are summarised in Table 1 and shown in Figures 2 and 3. In the X-rays, EXO 0748-676 shows a steady decrease by about 40 per cent over the $\simeq 9.5$ yr time span covering the observations (see also Parikh et al. 2020). In the UV band, such long-term variations are not clearly seen.

EXO 0748-676 was not detected in the UV during the first XMMNewton epoch, when the OM was used with the UVW1 filter. How-

10 The response for the $H S T / \mathrm{COS}$ acquisition image is not available, so we took the WFC3/UVIS F218W filter for this as a proxy. 


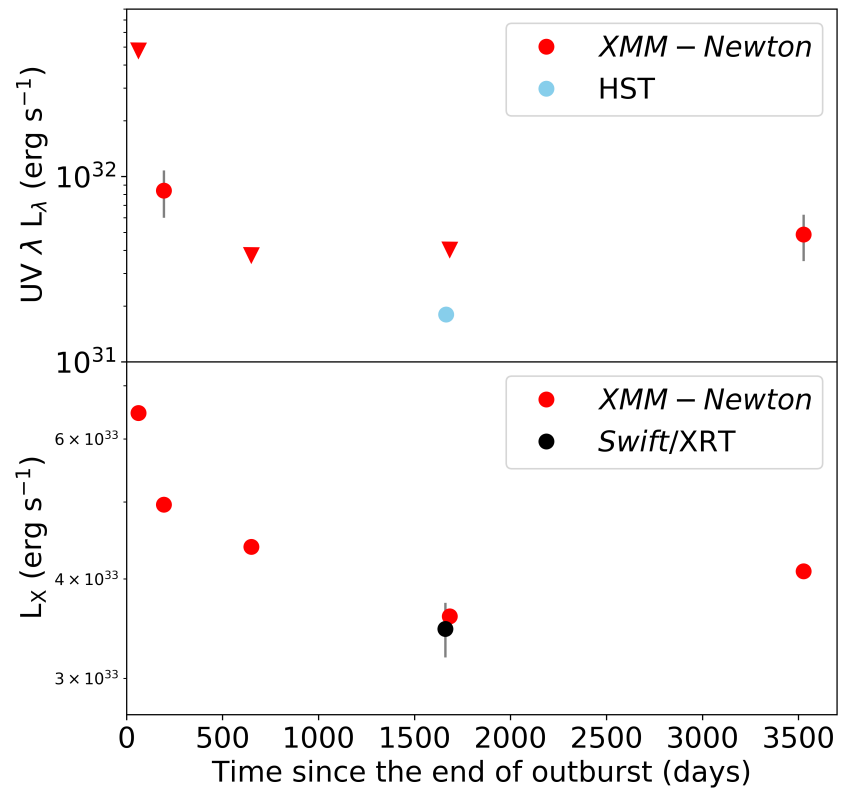

Figure 2. The top and bottom panels show the quasi-simultaneous evolution of the UV and X-ray luminosity of EXO 0748-676 during quiescence. The data from the quasi-simultaneous HST and Swift observations are shown in blue and black, respectively. The red data indicate those observations obtained using XMM-Newton. All upper limits are marked by downwardfacing triangles. We note that the plotted UV data do not take into account uncertainties on the UV spectral shape, which most strongly affects the HST data point (see Section A1); caution should therefore be taken in comparing this point to the XMM-Newton data (the first of which was taken with the UVW1 filter, the others with the U filter).

ever, the obtained upper limit is not constraining and much higher than the U-band luminosities measured at later times. During the second and last XMM-Newton epochs, EXO 0748-676 is detected in the $U$ filter at luminosities of $\simeq 8.5$ and $5 \times 10^{31} \mathrm{erg} \mathrm{s}^{-1}$, respectively, but with large errors; the two data points are consistent within $2 \sigma$ even without taking into account the additional uncertainty of the UV spectral shape in this band (see Section 3.2.4). The X-ray luminosity corresponding to these two data points differs by about 20 per cent. EXO 0748-676 is not detected in the U-band in the other two observations, but the obtained upper limits are not significantly smaller than the U-band luminosities inferred for the two detections. While the inferred luminosity from the HST acquisition image is a factor of a few lower than the U-band luminosities measured with $X M M$ Newton (see Table 1 and Figure 2), the unknown UV spectral shape introduces significant errors in this band (see Section 3.2.4). The available data thus does not indicate that there are strong variations in the UV emission of EXO 0748-676 over the $\simeq 9.5$-yr observing period. However, the uncertainties are large and variations of similar magnitude as seen in the X-rays ( $\simeq 40$ per cent) cannot be excluded.

Figure 3 also shows the X-ray versus UV luminosity for other NS and BH LMXBs observed in quiescence, as reported by Hynes \& Robinson (2012). It is clear from this plot that while the UV luminosity of EXO 0748-676 is in the same realm as that observed for other LMXBs, its X-ray luminosity, and hence its X-ray/UV luminosity ratio, is much larger than that of any of the other systems.

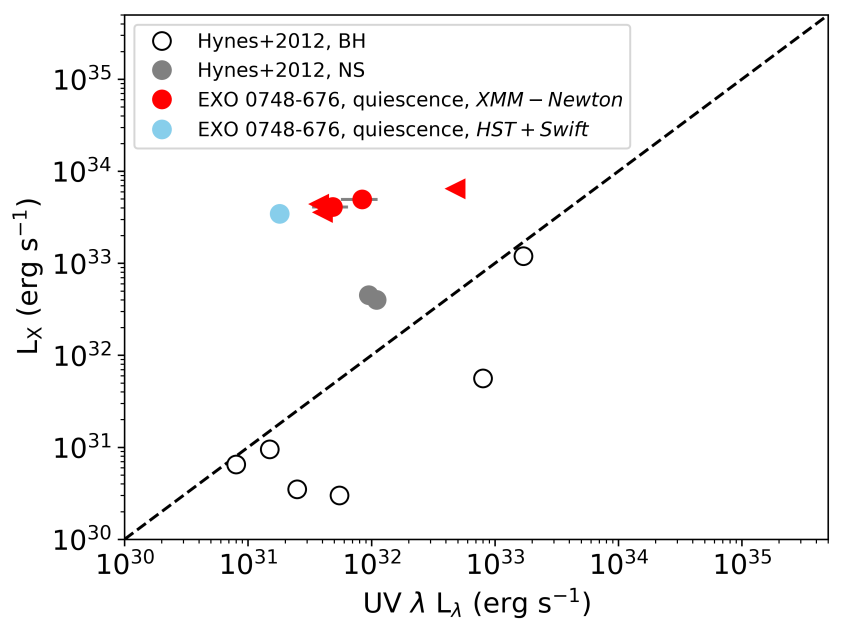

Figure 3. The observed X-ray versus UV luminosity of EXO 0748-676 during six quiescent epochs is shown. Five epochs were obtained using $X M M$ Newton (shown in red). One epoch was observed using HST and the Swift, and is shown in blue. We note that these data points do not take into account the unknown UV spectral shape (see Section A1). We compare EXO 0748676 to eight other quiescent LMXBs studied in Hynes \& Robinson (2012). The six systems hosting BHs are shown by open black circles and represent GRO J0422+32 (V518 Per), MM Vel, XTE J118-40, A0620-00, GU Mus, and V404 Cyg (listed in ascending order of the UV luminosity). The two NS systems are shown by filled grey circles and correspond to Aql X-1 and Cen X-4. All upper limits are marked by triangles facing the relevant direction.

\section{DISCUSSION}

Investigating quiescent spectra of LMXBs allows us to understand the accretion behaviour in these systems at low luminosities. In addition, quiescent studies of accretion-heated NS crusts in LMXBs provide us with the opportunity to probe the properties of dense matter physics. We have examined the crust-cooling source EXO 0748-676 in quiescence. Early in quiescence, this source has behaved like a typical crust-cooling source. However, recently obtained observations indicate an unexpected temperature rise (see Section 1.1). This rise in temperature may be a result of increased low-level accretion in quiescence, although studies of the X-ray spectra indicate that this is likely not the case (see Parikh et al. 2020, for more details). Here we have further investigated the possibility of ongoing accretion in quiescence in EXO 0748-676 by examining the quiescent FUV spectrum and several epochs of (quasi-)simultaneous X-ray and NUV data.

\subsection{The quiescent FUV spectrum}

Due to the inherently low quiescent FUV flux of EXO 0748-676, all FUV spectroscopic data had to be stacked to increase the signal to noise and hence no phase-resolved study could be performed. We do not detect FUV continuum emission from the source, but there is one prominent emission line detected, $C_{\text {IV }}(\simeq 1550 \AA)$, for which we measure a width of $\gtrsim 18.2 \AA\left(\gtrsim 3523 \mathrm{~km} \mathrm{~s}^{-1}\right)$.

The FUV spectrum of this source obtained during the outburst also shows a prominent $\mathrm{C}$ IV line (alongside other emission lines), for which we measure a width of $\simeq 19.5 \AA$. Tomograms made using the outburst data, presented in Pearson et al. (2006), show that the $\mathrm{C}$ IV emission may arise from the accretion disc, the accretion impact stream, and the irradiated donor star. Our obtained lower limit on the width of this line in quiescence is close to the value measured during outburst. This could suggest that the line is produced at a 
Table 1. Log of the (quasi-)simultaneous UV and X-ray observations of EXO 0748-676 in quiescence.

\begin{tabular}{lllcclcc}
\hline Epoch & MJD & Observatory & UV band & $\begin{array}{c}\text { Central UV } \\
\text { wavelength }^{a}(\AA)\end{array}$ & $\begin{array}{l}\mathrm{UV} \lambda \mathrm{L}_{\lambda} \\
\left(\times 10^{31} \mathrm{erg} \mathrm{s}^{-1}\right)\end{array}$ & $\begin{array}{l}\text { Unabsorbed } \mathrm{L}_{X} \\
\left(\times 10^{32} \mathrm{erg} \mathrm{s}^{-1}\right)\end{array}$ & $\begin{array}{l}\mathrm{L}_{X} / \mathrm{L}_{\mathrm{UV}} \\
\text { ratio }\end{array}$ \\
\hline 1 & 54776.4 & XMM-Newton & $\mathrm{UVW1}$ & 2910 & $<47.8$ & $64.7 \pm 0.5$ & $>13.5$ \\
2 & 54908.0 & XMM-Newton & $\mathrm{U}$ & 3400 & $8.4 \pm 2.4$ & $49.6 \pm 0.3$ & $\simeq 59.1$ \\
3 & 55364.2 & XMM-Newton & $\mathrm{U}$ & 3400 & $<3.8$ & $43.9 \pm 0.3$ & $>115.5$ \\
4 & $55376.5^{b}$ & HST \& Swift & NUV & 2300 & $\simeq 1.8$ & $34.7 \pm 2.6$ & $\simeq 243.9$ \\
5 & 56397.2 & XMM-Newton & $\mathrm{U}$ & 3400 & $<4.0$ & $35.9 \pm 0.2$ & $>89.8$ \\
6 & 58240.3 & XMM-Newton & $\mathrm{U}$ & 3400 & $4.9 \pm 1.4$ & $40.9 \pm 0.2$ & $\simeq 83.5$ \\
\hline
\end{tabular}

We have assumed $D=7.4 \mathrm{kpc}$ and $E(B-V)=0.06$. We caution that the UV luminosities quoted here assume a

flat spectral shape; see Section 3.2.4 for a discussion on the impact of the unknown spectral shape.

${ }^{a}$ Obtained from https://www.swift.ac.uk/analysis/uvot/filters.php.

${ }^{b}$ The MJD for this epoch has been determined by averaging the MJD of the HST and Swift observations

similar location. Studies of other LMXBs suggest their quiescent UV emission may arise from the heated inner edge of the accretion disc, the accretion impact stream, and the irradiated donor star (e.g., Froning et al. 2011; Hynes \& Robinson 2012; Cackett et al. 2013a; Bernardini et al. 2013a). In the following we investigate what the most likely origin of the quiescent UV emission is for EXO 0748-676.

\subsection{No disc/accretion stream in quiescence?}

Outburst studies of EXO 0748-676 in the X-ray indicated the presence of 'dipping' features likely due to the presence of an ionised absorber (e.g., Sidoli et al. 2005). Psaradaki et al. (2018) examined the high-resolution outburst spectra during the eclipses using the Reflection Grating Spectrometer (RGS) on board XMM-Newton. Their study found emission lines which may be due to clumps produced by the presence of the accretion stream as it impacts the disc. To investigate whether the $C_{\text {IV }}$ line we detect from the source in quiescence may arise from such clumps, we extrapolated their outburst model to the FUV. We have further extrapolated this to quiescence by using the RGS data obtained during early quiescence (see Díaz Trigo et al. 2011, for details). However, no significant emission lines were present in these data and, therefore, the study carried out for the outburst spectrum could not be extended to quiescence. Furthermore, no dipping features have been observed in the X-ray during quiescence, which instead exhibited eclipses with sharp ingresses and egresses (Díaz Trigo et al. 2011; Degenaar et al. 2014). This raises the question whether the obscuring structure, i.e., the disc and/or accretion stream impact point, is present in quiescence.

Optical spectroscopy studies of EXO 0748-676, carried out $\simeq 1 \mathrm{yr}$ into quiescence (Ratti et al. 2012), showed no indications of the presence of an accretion disc (as discerned from the optical line emission). It is worth noting though, that this study was carried out early in quiescence, while our HST observations were obtained $\simeq 3.5 \mathrm{yr}$ later and the disc may have built up over time. Such a buildup of the accretion disk may have been observed for another edge-on crust-cooling source, MXB 1659-29. For that source, this scenario was proposed based on a possible increase in the absorption column density as inferred from X-ray spectral fitting (see Cackett et al. 2013b, for details). ${ }^{11}$ However, as argued above it is not obvious that this was the case for EXO 0748-676, so we continue to explore other possibilities for the origin of is UV emission.

11 This change was observed $\simeq 11$ yr into quiescence and $\simeq 3$ yr before MXB 1659-29 was seen to enter a new outburst (Negoro et al. 2015).

\subsection{Irradiation of the companion star}

Although no evidence of an accretion disc was found by Ratti et al. (2012), they observed variations at different orbital phases indicating that the irradiated face of the companion star was emitting in the optical. This raises the possibility that the quiescent UV radiation may also arise due to the irradiation of the companion by the central X-ray source. However, the observed C IV emission line is much broader than the observed optical lines. We measure a lower limit of $\gtrsim 3523 \mathrm{~km} \mathrm{~s}^{-1}$, whereas the reported widths of the optical spectral lines are $\simeq 300-400 \mathrm{~km} \mathrm{~s}^{-1}$ (see table 1 of Ratti et al. 2012). ${ }^{12}$

Based on their optical study in quiescence, Ratti et al. (2012) suggest that EXO 0748-676 may be a black widow system, in which the pulsar wind interacts with the companion star, slowly obliterating it. ${ }^{13}$ This scenario would require the NS in EXO 0748-676 to turn on as a radio pulsar in quiescence. It may then be similar to the handful of known transitional millisecond radio pulsars (tMSRPs) that appear to switch between radio pulsar states and low luminosity LMXB-like states in which an accretion disc is detected in the system in the optical band.

The optical lines in the spectrum of PSR J1023+0038, one of the tMSRPs, arise from irradiation of the companion star and are narrow (McConnell et al. 2015), like the optical lines of EXO 0748-676 in quiescence (Ratti et al. 2012). The difference in line width makes it unlikely that the C IV emission line observed for EXO 0748-676 arises due to irradiation of the companion star, but the radio pulsar scenario does pose another possibility for its origin.

\subsection{Pulsar wind or optically thin accretion disc}

Interestingly, the UV lines of PSR J1023+0038 are also very broad $\left(\simeq 2000-3000 \mathrm{~km} \mathrm{~s}^{-1}\right)$. Since the accretion disc in this system is truncated very far away from the NS, it was proposed that these lines are produced by the pulsar wind ionising material from the companion star (i.e., the lines would then be broadened by the velocity of the wind; Hernández Santisteban 2016). Possibly a similar physical

12 We note that the $\mathrm{C}$ IV line is actually a doublet, separated by $\simeq 500 \mathrm{~km} \mathrm{~s}^{-1}$, so it is possible that in our low signal to noise data two lines are merged into one. Nevertheless, the lines would still need to be significantly broader than the lines in the optical quiescent spectrum presented in Ratti et al. (2012).

13 We note that the constraints on the mass of the donor star yield $>0.11 \mathrm{M}_{\odot}$ for a 'canonical' NS mass of $1.4 \mathrm{M}_{\odot}$ (see Bassa et al. 2009, and references therein), which means that should EXO 0748-676 be an active pulsar, it would be classed as a redback, like the tMSRPs, rather than a black widow. 
scenario can explain the broadness of the C IV emission line in EXO 0748-676.

Inspecting the red wing of the airglow emission line around $\simeq 1220 \AA$ in the quiescent UV spectrum of EXO 0748-676 suggests that there may be an excess corresponding to the $\mathrm{Nv}$ emission line that was seen in outburst (Pearson et al. 2006). We cannot confidently constrain its presence, but if this line is there, this could lend support for the pulsar wind scenario. This mechanism has also been invoked to explain the UV excess in two systems hosting tMSRPs (Rivera Sandoval et al. 2018). Nevertheless, this has only been shown for a small sample of sources and similar studies cannot be carried out for EXO 0748-676 as its continuum emission is not detected.

Another possibility, which does not require invoking EXO 0748676 turns on as a radio pulsar in quiescence, is that material from the companion star is ionised by a geometrically thick, optically thin quiescent accretion flow. If such a flow is present in this system, we might expect material to accrete on to the NS (e.g., Chakrabarty et al. 2014; D'Angelo et al. 2015). However, there are no signs of ongoing accretion in EXO 0748-676.

\subsection{The (lack of) X-ray/UV correlation}

In addition to FUV spectra, we have also examined several epochs of quasi-simultaneous X-ray and NUV data in quiescence. If the UV emission arises somewhere in a quiescent accretion flow that reaches the NS, a correlation between the UV and X-ray emission would be expected. Taking into account the uncertainty of the UV spectral shape, we find no evidence for significant changes in the UV emission during quiescence, although the uncertainties are so large that changes of similar magnitude as seen in the X-rays can be hidden within the errors.

Despite that we cannot firmly determine if the X-ray and UV emission change in tandem, the X-ray/UV luminosity ratio gives additional information. We determined the ratio of quiescent X-ray to NUV emission to be $L_{\mathrm{X}} / L_{\mathrm{UV}} \gtrsim 100$. Since the UV luminosity of EXO 0748-676 is of similar magnitude as that of other LMXBs, this high ratio would suggest that it is considerably more X-ray bright than other systems (see Figure 3). In particular, the two NS LMXBs in the sample of Hynes \& Robinson (2012), Cen X-4 and Aql X-1, exhibit $L_{\mathrm{X}} / L_{\mathrm{UV}} \simeq 10$. In Cen $\mathrm{X}-4$, there is compelling evidence that the intrinsic NUV emission comes from the accretion flow (e.g., Cackett et al. 2013a; Bernardini et al. 2013a), and that matter accretes onto the surface of the NS even at these low luminosities (e.g., Chakrabarty et al. 2014; D'Angelo et al. 2015). The same might be true for Aql X-1, which exhibits X-ray variability of several magnitudes in quiescence (e.g., Rutledge et al. 2001; Campana \& Stella 2003; Cackett et al. 2011; Coti Zelati et al. 2014a). The fact that EXO 0748-676 has a similar UV luminosity as these systems but is much brighter in the X-rays, would then suggest that its X-ray emission is not (predominantly) powered by low-level accretion.

In conclusion, our study of (quasi-)simultaneous UV and X-ray observations of EXO 0748-676 in quiescence hints that the observed broad UV emission line arises from ionisation of material from the companion star, possibly by a pulsar wind. Similar to previous optical and X-ray studies, our combined UV/X-ray analysis does not reveal evidence for the presence of a quiescent accretion stream, nor that the high quiescent X-ray emission of EXO 0748-676 arises from ongoing low-level accretion. Our results thus seem to be in favor of explaining the bulk of the quiescent X-ray emission of EXO 0748676 as cooling of the NS crust. This implies that such studies can be used to infer the properties of the crust and core of this NS (e.g., Degenaar et al. 2011a; Parikh et al. 2020).

\section{ACKNOWLEDGEMENTS}

The authors are very grateful to the referee, Craig Heinke, for a valuable report that helped improve this manuscript. AP, ND and JVHS are supported by a Vidi grant awarded to ND by the Netherlands Organization for Scientific Research (NWO). IP and EC are supported by the Vidi grant 639.042.525. JVHS acknowledges funds from a Science and Technology Facilities Council grant ST/R000824/1 research fellowship. Further support for HST program GO-13108 was provided by NASA through a grant from the STScI.

\section{DATA AVAILABILITY}

The data underlying this article are available in Zenodo, at https://doi.org/10.5281/zenodo.3908291. The datasets were derived from sources in the public domain: https: / / archive.stsci. edu/hst/search.php, http://nxsa.esac.esa.int/ nxsa-web/\#home, and https://heasarc.gsfc.nasa. gov/cgi-bin/w3Browse/swift.pl.

\section{REFERENCES}

Astropy-Specutils Development Team 2019, Specutils: Spectroscopic analysis and reduction (ascl:1902.012)

Allard F., Homeier D., Freytag B., 2012, Phil. Trans. R. Soc. A, 370, 2765

Bassa C. G., Jonker P. G., Steeghs D., Torres M. A. P., 2009, MNRAS, 399, 2055

Bernardini F., Cackett E., Brown E., D’Angelo C., Degenaar N., Miller J., Reynolds M., Wijnands R., 2013a, MNRAS, 436, 2465

Brown E. F., Cumming A., 2009, ApJ, 698, 1020

Brown E. F., Bildsten L., Rutledge R. E., 1998, ApJ Letters, 504, L95

Burrows D., et al., 2005, Space Science Reviews, 120, 165

Cackett E., Fridriksson J., Homan J., Miller J., Wijnands R., 2011, MNRAS, 414, 3006

Cackett E., Brown E., Degenaar N., Miller J., Reynolds M., Wijnands R., 2013a, MNRAS, 433, 1362

Cackett E., Brown E., Cumming A., Degenaar N., Fridriksson J., Homan J., Miller J., Wijnands R., 2013b, ApJ, 774, 131

Campana S., Stella L., 2003, ApJ, 597, 474

Cardelli J., Clayton G., Mathis J., 1989, ApJ, 345, 245

Carnall A. C., 2017, arXiv e-prints, p. arXiv:1705.05165

Chakrabarty D., et al., 2014, ApJ, 797, 92

Cheng Z., Mendez M., Diaz-Trigo M., Costantini E., 2017, MNRAS, 471, 2605

Coti Zelati F., Campana S., D’Avanzo P., Melandri A., 2014a, MNRAS, 438, 2634

Cumming A., Brown E. F., Fattoyev F. J., Horowitz C. J., Page D., Reddy S., 2017, Phys. Rev. C, 95, 025806

D’Angelo C. R., Fridriksson J. K., Messenger C., Patruno A., 2015, MNRAS, 449, 2803

Degenaar N., Wijnands R., 2012, MNRAS, 422, 581

Degenaar N., et al., 2009a, MNRAS, 396, L26

Degenaar N., et al., 2011a, MNRAS, 412, 1409

Degenaar N., Brown E. F., Wijnands R., 2011b, MNRAS, 418, L152

Degenaar N., et al., 2014, ApJ, 791, 47

Deufel B., Dullemond C. P., Spruit H. C., 2001, A\&A, 377, 955

Díaz Trigo M., Boirin L., Costantini E., Méndez M., Parmar A., 2011, A\&A, 528,150

Done C., Gierliński M., Kubota A., 2007, A\&ARv, 15, 1

Dubus G., Hameury J.-M., Lasota J.-P., 2001, A\&A, 373, 251 
Ely J., et al., 2015, in IAU General Assembly. p. 2255542

Fridriksson J. K., Homan J., Wijnands R., Cackett E. M., Altamirano D., Degenaar N., Brown E. F., et al., 2011, ApJ, 736, 162

Froning C., et al., 2011, ApJ, 743, 26

Froning C. S., Maccarone T. J., France K., Winter L., Robinson E. L., Hynes R. I., Lewis F., 2014, ApJ, 780, 48

Galloway D., Özel F., Psaltis D., 2008, MNRAS, 387, 268

Gehrels N., et al., 2004, ApJ, 611, 1005

Gilfanov M., 2010, X-Ray Emission from Black-Hole Binaries. p. 17, doi:10.1007/978-3-540-76937-8_2

Green J., et al., 2012, ApJ, 744, 60

Haensel P., Zdunik J., 1990, A\&A, 227, 431

Haensel P., Zdunik J., 2008, A\&A, 480, 459

Hameury J. M., 2020, AdSpR, 66, 1004

Heinke C. O., Rybicki G. B., Narayan R., Grindlay J. E., 2006, ApJ, 644, 1090

Hernández Santisteban J. V., 2016, PhD thesis, e-print https://eprints.soton.ac.uk/404707/

Holland et al. S. T., 2012, Cosmic Origins Spectrograph Instrument Handbook for Cycle 21 v. 5.0

Hynes R. I., Haswell C. A., 1999, MNRAS, 303, 101

Hynes R., Jones E., 2008, ATel, 1816

Hynes R., Robinson E., 2012, ApJ, 749, 3

Hynes R. I., Robinson E. L., Bitner M., 2005, ApJ, 630, 405

Hynes R. I., Horne K., O’Brien K., Haswell C. A., Robinson E. L., King A. R., Charles P. A., Pearson K. J., 2006, ApJ, 648, 1156

Joseph C. L., et al., 1995, Performance results of the STIS flight MAMA detectors. pp 248-259, doi:10.1117/12.218636

Lasota J.-P., 2001, New Astronomy Reviews, 45, 449

Mason K. O., et al., 2001, A\&A, 365, L36

McConnell O., Callanan P. J., Kennedy M., Hurley D., Garnavich P., Menzies J., 2015, MNRAS, 451, 3468

Narayan R., Yi I., 1994, ApJ, 428, L13

Negoro H., Furuya K., Ueno S., Tomida H., Nakahira S., Kimura M., Ishikawa M., et al., 2015, ATel, 7943

Ootes L. S., Wijnands R., Page D., Degenaar N., 2018, MNRAS, 477, 2900

Page D., Reddy S., 2013, Phys. Rev. Lett., 111, 241102

Parikh A. S., et al., 2017, MNRAS, 466, 4074

Parikh A. S., Wijnands R., Homan J., Degenaar N., Wolvers B., Ootes L. S., Page D., 2020, A\&A, 638, L2

Parmar A., White N., Giommi P., Haberl F., Pedersen H., Mayor M., 1985, IAU Circ., 4039

Parmar A., White N., Giommi P., Gottwald M., 1986, ApJ, 308, 199

Pearson K. J., et al., 2006, ApJ, 648, 1169

Psaradaki I., Costantini E., Mehdipour M., Díaz Trigo M., 2018, A\&A, 620, A129

Ratti E., Steeghs D., Jonker P., Torres M., Bassa C., Verbunt F., 2012, MNRAS, 420,75

Rivera Sandoval L. E., et al., 2018, MNRAS, 476, 1086

Roming P. W. A., et al., 2005, Space Sci. Rev., 120, 95

Rutledge R., Bildsten L., Brown E., Pavlov G., Zavlin V., 2001, ApJ, 559, 1054

Shakura N. I., Sunyaev R. A., 1973, A\&A, 500, 33

Shternin P. S., Yakovlev D. G., Haensel P., Potekhin A. Y., 2007, MNRAS, 382, L43

Sidoli L., Parmar A. N., Oosterbroek T., 2005, A\&A, 429, 291

Steiner A. W., 2012, Phys. Rev. C, 85, 055804

Strüder L., et al., 2001, A\&A, 365, L18

Tody D., 1986, The IRAF Data Reduction and Analysis System. p. 733, doi: $10.1117 / 12.968154$

Torres M., Jonker P., Steeghs D., Seth A., 2008, ATel, 1817

Turlione A., Aguilera D. N., Pons J. A., 2015, A\&A, 577, A5

Turner M. J., et al., 2001, A\&A, 365, L27

Wijnands R., Miller J. M., Markwardt C., Lewin W. H., van der Klis M., 2001, ApJ Letters, 560, L159

Wijnands R., Degenaar N., Padilla M. A., Altamirano D., Cavecchi Y., Linares M., Bahramian A., Heinke C., 2015, MNRAS, 454, 1371
Wijnands R., Degenaar N., Page D., 2017, Journal of Astrophysics and Astronomy, 38, 49

Wolff M., Becker P., Ray P., Wood K., 2005, ApJ, 632, 1099

Wolff M., Ray P., Wood K., 2008a, ATel, 1736

Wolff M., Ray P., Wood K., Wijnands R., 2008b, ATel, 1812

Wolff M., Ray P., Wood K., Hertz P., 2009, ApJS, 183, 156

Yan Z., Yu W., 2015, ApJ, 805, 87

\section{APPENDIX A: UV SPECTRAL SHAPE CORRECTIONS}

This paper has been typeset from a $\mathrm{T}_{\mathrm{E}} \mathrm{X} / \mathrm{L} \mathrm{T}_{\mathrm{E}} \mathrm{X}$ file prepared by the author. 

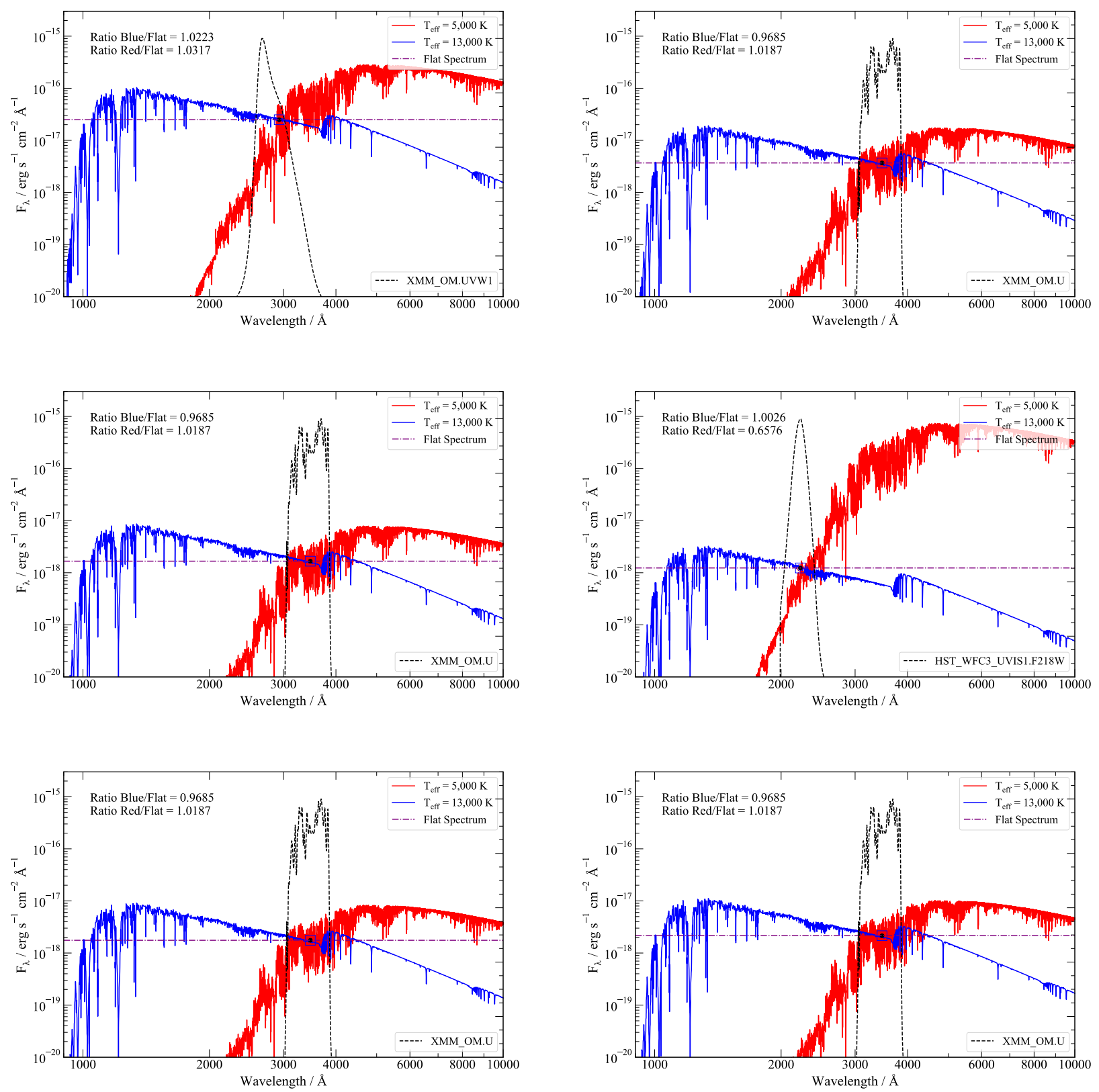

Figure A1. Estimates of the effect of the unknown UV spectral shape on the inferred UV luminosity. For each observation we show the measured UV flux (upper limit) as the black data point, while the blue and red curves represent stellar-atmosphere spectra with effective temperatures of $13000 \mathrm{~K}$ and $5000 \mathrm{~K}$, respectively. The black dashed curves show the different filter transmissions. Printed in the top left are the ratios of the integrated fluxes for these models compared to a flat distribution, indicated by the dash-dotted horizontal purple lines, over the filter passband. These plots show that the unknown spectral shape has a relatively minor impact on the calculated U-band luminosity (of a few per cent), but has a larger impact for bluer filters. 